\title{
Perfil sociodemográfico e clínico de pessoas em tratamento quimioterápico: subsídios para o gerenciamento em enfermagem
}

Sociodemographic and clinical profile of people undergoing chemotherapy: support for nursing management

Perfil sociodemográfico y clínico de personas en tratamiento quimioterápico: ayudas para el gerenciamiento en enfermería

Marcelle Miranda da Silva', Jaciane Alexandre da Silva², Lidiane Orineu Esteves³, Maria Gefé da Rosa Mesquita, Marluci Andrade Conceição Stipp ${ }^{5}$, Sabrina da Costa Machado Duarte ${ }^{6}$

\footnotetext{
${ }^{1}$ Enfermeira, Doutora em Enfermagem. Professora Adjunta da Escola de Enfermagem Anna Nery da Universidade Federal do Rio de Jan eiro (EEAN/UFRJ). Rio de Janeiro, RJ, Brasil. E-mail: mmarcelle@ig.com.br.

${ }^{2}$ Enfermeira. Residente em Enfermagem do Instituto de Hematologia Arthur de Siqueira Cavalcanti (HEMORIO). Rio de Janeiro, RJ, Brasil. E-mail: jacianealexandre@gmail.com.

${ }^{3}$ Enfermeira. Rio de Janeiro, RJ, Brasil. E-mail: lidianeoesteves@yahoo.com.br.

${ }^{4}$ Enfermeira, Doutora em Enfermagem. Professora Adjunta da EEAN/UFRJ. Rio de Janeiro, RJ, Brasil. E-mail: mariagefe@gmail.com.

${ }^{5}$ Enfermeira, Doutora em Enfermagem. Professora Associada da EEAN/UFRJ. Rio de Janeiro, RJ, Brasil. E-mail: marlustipp@gmail.com.

${ }^{6}$ Enfermeira, Mestre em Enfermagem. Discente do Programa de Pós-Graduação em Enfermagem, nível Mestrado, da EEAN/UFRJ. Professora Assistente da EEAN/UFRJ. Rio de Janeiro, RJ, Brasil. E-mail: inamachado@globo.com.
}

\section{RESUMO}

Objetivou-se caracterizar o perfil sociodemográfico e clínico das pessoas assistidas no ambulatório de quimioterapia, e evidenciar as contribuições do conhecimento deste para o gerenciamento das ações de enfermagem. Estudo não experimental, descritivo, documental, quantitativo, realizado no Hospital Universitário Clementino Fraga Filho com 87 prontuários investigados entre outubro e dezembro de 2010 . Dos resultados: $59,7 \%$ do sexo feminino; grande número de pessoas idosas; $59,8 \%$ casados; $38 \%$ com ensino fundamental incompleto; 35,6\% com tumor na mama; náuseas e vômitos como principais queixas; 36,9\% em tratamento paliativo; $94,3 \%$ utilizando a via endovenosa; $43,7 \%$ dos prontuários sem registros de intercorrências. As informações clínicas e não clínicas contribuem para diferentes aspectos gerenciais. No gerenciamento do cuidado de enfermagem podem guiar o processo de tomada de decisão e ação, contribuindo para o cuidado integral, em consonância com a realidade de cada indivíduo, de forma contextualizada e humanizada. A complexidade e escassez de estudos demandam novas pesquisas.

Descritores: Neoplasias; Antineoplásicos; Administração dos Cuidados ao Paciente; Gerência; Enfermagem.

\section{ABSTRACT}

The objective was to characterize the sociodemographic and clinical profile of people assisted in a chemotherapy outpatient clinic, and give evidence of the contributions that this knowledge provides to managing nursing activities. This non-experimental, descriptive, documental, quantitative study was performed at the Clementino Fraga Filho University Hospital, with 87 medical records, investigated between October and December of 2010 . The results show that: $59.7 \%$ are female; high number of elderly people; $59.8 \%$ married; $38 \%$ with an incomplete primary education; $35.6 \%$ with breast tumors; nausea and vomiting as the major complaints; $36.9 \%$ undergoing palliative care; $94.3 \%$ using the intravenous route; $43.7 \%$ of the records without any notes regarding complications. The clinical and non-clinical information contribute for diverse managerial aspects. In the management of nursing care this information can guide in decision making and in planning nursing activities, thus contributing with comprehensive care, in agreement with the situation of each individual in a contextualized and humanized way. The complexity and scarcity of studies demand further research.

Descriptors: Neoplasms; Antineoplastic Agents; Patient Care Management; Management; Nursing.

\section{RESUMEN}

Se objetivó caracterizar el perfil sociodemográfico y clínico de personas atendidas en ambulatorio de quimioterapia, y evidenciar las contribuciones del conocimiento de éste para el gerenciamiento de acciones de enfermería. Estudio no experimental, descriptivo, documental, cuantitativo, realizado en Hospital Universitario Clementino Fraga Filho, con 87 historias clínicas, investigadas de octubre a diciembre 2010. De los resultados: 59,7\% de sexo femenino; alto número de ancianos; $59,8 \%$ casados; $38 \%$ con enseñanza primaria incompleta; $35,6 \%$ con tumor mamario; náuseas y vómitos como principales quejas; $36,9 \%$ en tratamiento paliativo; $94,3 \%$ utilizando vía endovenosa; $43,7 \%$ de historias clínicas sin registros de variaciones. Las informaciones clínicas y no clínicas contribuyen con diferentes aspectos gerenciales. En el gerenciamiento del cuidado de enfermería pueden orientar el proceso de toma de decisiones y acciones, contribuyendo al cuidado integral, en consonancia con la realidad del individuo, de forma contextualizada y humanizada. La complejidad y escasez de estudios demandan nuevas investigaciones.

Descriptores: Neoplasias; Antineoplásicos; Manejo de Atención al Paciente; Gerencia; Enfermería. 


\section{INTRODUÇÃO}

O câncer representa a segunda causa de morte por doença na população brasileira desde 2003, sendo responsável por, aproximadamente, $17 \%$ dos óbitos de causas conhecidas, notificados em 2007 no Sistema de Informações sobre Mortalidade do Instituto Nacional de Câncer (INCA). As estimativas para o ano 2012, válidas também para 2013, publicadas pelo INCA, referem 518.510 casos novos de câncer no país, incluindo os casos de pele não melanoma ${ }^{(1)}$.

Diante dos elevados índices de morbi-mortalidade por câncer, as medidas para o diagnóstico precoce aumentam a possibilidade de controle e de cura da doença. Vários aspectos podem facilitar o reconhecimento precoce de sinais e sintomas do câncer por parte dos profissionais da área da saúde, bem como podem contribuir para o melhor gerenciamento de suas ações em prol da qualidade da assistência, direcionando a atuação em resposta às demandas e realidades específicas. Destacam-se as variáveis que dizem respeito aos determinantes sociais da saúde na população afetada pela doença, o que pode representar o desenho de um perfil sociodemográfico, englobando, neste caso, fatores sociais, econômicos, dentre outros ${ }^{(2)}$. Estes influenciam os fatores de risco, a ocorrência de problemas de saúde e o gerenciamento dos mesmos por parte dos envolvidos, o que ressalta a importância de sua conjugação com variáveis clínicas.

Dessa forma, o conhecimento das variáveis prevalentes em grupos de pessoas acometidas pelo câncer tem a intenção de reunir subsídios para o planejamento de diversas ações, com diferentes metas, níveis de impacto e alcance. São exemplos: atacar as causas do câncer, com cunho preventivo, bem como das iniquidades em matéria de saúde; e funcionar como ferramentas para o gerenciamento, seja do ambiente, dos serviços, dos riscos ou do próprio cuidado.

Destaca-se no presente estudo a vertente do gerenciamento do cuidado de enfermagem a partir da investigação do perfil sociodemográfico e clínico das pessoas em tratamento quimioterápico ambulatorial. $O$ destaque para a quimioterapia decorre desta ser a terapêutica instituída na maioria das pessoas diagnosticadas com câncer, sendo o tratamento de escolha quando há acometimento do sistema hematopoético e nos casos dos tumores sólidos, que apresentam ou não metástases regionais ou à distância ${ }^{(3)}$.
Os profissionais responsáveis pela administração da quimioterapia compõem a equipe de enfermagem, sendo o cuidado gerenciado pelo enfermeiro, e respaldado pela Resolução $n^{\circ}$ 210/1998 do Conselho Federal de Enfermagem (COFEN), que dispõe sobre a atuação dos profissionais de enfermagem que trabalham com quimioterápicos antineoplásicos ${ }^{(4)}$. Logo, no contexto do ambulatório de quimioterapia, as informações do perfil podem ser capazes de gerar subsídios para as atividades da equipe de enfermagem, uma vez que o gerenciamento do cuidado envolve planejamento, organização, supervisão, execução e avaliação de todas as atividades de enfermagem na assistência à pessoa ${ }^{(5)}$.

$O$ atendimento das necessidades de cuidado das pessoas em tratamento quimioterápico é facilitado por meio do adequado gerenciamento do cuidado, considerando a abordagem individual, integral e humanizada, em prol da prevenção e do controle de possíveis agravos decorrentes da terapêutica.

No cumprimento das atividades do gerenciamento do cuidado de enfermagem, o enfermeiro precisa desenvolver habilidades e competências para o melhor uso dos recursos disponíveis. Estes incluem o reconhecimento do perfil das pessoas atendidas, de forma a ter atenção voltada aos possíveis eventos adversos da quimioterapia, prognósticos e fatores de risco associados à terapêutica, levando em consideração todos os aspectos que podem interferir na qualidade de vida das mesmas no decorrer do tratamento(6).

Em assim sendo, o estudo objetivou caracterizar o perfil sociodemográfico e clínico das pessoas assistidas no ambulatório de quimioterapia, e evidenciar as contribuições do conhecimento deste para o gerenciamento das ações de enfermagem.

O estudo se justifica pela forte e crescente evidência da relação entre aspectos não clínicos e o estado de saúde das pessoas, direcionando desde ações específicas relativas ao cuidado de enfermagem no decorrer do tratamento quimioterápico, além de poder contribuir para vinculações mais gerais que envolvem o desenvolvimento do câncer e as características específicas do contexto social das pessoas ${ }^{(2)}$.

Além disso, destaca-se a escassez de estudos que abordam esta temática, não tendo sido encontrado nenhum artigo científico no contexto nacional na busca realizada na Biblioteca Virtual em Saúde, no período entre 2008 a 2012, a partir dos descritores neoplasias e 
antineoplásicos. O necessário investimento em pesquisa decorre da representatividade epidemiológica e etiologia multifatorial do câncer, sendo importante melhor caracterizar o perfil das pessoas atendidas com este diagnóstico, de modo a remeter a problemática nacional ao contexto global, e direcionar condutas nos diferentes níveis de atenção à saúde(7). Logo, a relação entre variáveis clínicas e não clínicas visa contribuir para a possibilidade de prevenção da doença, diagnóstico precoce, prevenção e controle das morbidades e dos eventos adversos da terapêutica, redução dos custos dos cofres públicos e da iniciativa privada relacionados às terapias em fases mais avançadas da doença, o que engloba diferentes aspectos gerenciais.

\section{MÉTODO}

Estudo não experimental, descritivo, documental, de abordagem quantitativa, em que os dados foram coletados por meio da consulta aos prontuários das pessoas em tratamento quimioterápico ambulatorial. 0 cenário para coleta dos dados foi o ambulatório de quimioterapia do Hospital Universitário Clementino Fraga Filho (HUCFF), hospital público, da esfera federal, universitário, localizado no município do Rio de Janeiro Brasil. Este apresenta importante representatividade no que se refere ao atendimento à pessoa com câncer no âmbito do Sistema Único de Saúde (SUS), sendo um Centro de Assistência de Alta Complexidade em Oncologia (CACON).

De acordo com a Política Nacional de Atenção Oncológica, o CACON compreende um hospital com condições técnicas, instalações físicas, equipamentos e recursos humanos adequados à prestação de assistência especializada de alta complexidade para o diagnóstico definitivo e tratamento de todos os tipos de câncer ${ }^{(8)}$.

Os dados foram coletados no período da manhã e da tarde durante os meses de outubro a dezembro de 2010. A coleta foi realizada pela aluna de iniciação científica e por mais um membro do grupo de pesquisadores no arquivo médico do referido hospital e no próprio setor de quimioterapia. A aluna inserida no projeto recebeu treinamento por parte da pesquisadora responsável para que houvesse aplicação do instrumento de forma uniformizada, garantindo a correta coleta das informações. Foi realizado um pré-teste para assegurar validade e precisão do instrumento ${ }^{(9)}$.
O instrumento para coleta dos registros nos prontuários foi composto por questões fechadas buscando-se traçar o perfil sociodemográfico, conforme os determinantes sociais da saúde, e clínico das pessoas atendidas. Foram destacadas as variáveis demográficas e sociais como sexo, idade, estado civil, estrutura familiar, educação e ocupação, bem como variáveis relacionadas à doença e ao tratamento quimioterápico, tais como: tipo de câncer, proposta do tratamento, estadiamento, evidência de metástase, principais queixas e complicações, e via de administração do quimioterápico.

Os critérios de inclusão da amostra foram: prontuários de pessoas com idade mínima de 18 anos, acometidas por câncer e em tratamento quimioterápico ambulatorial. A amostra foi constituída por prontuários de 87 dos 200 usuários totais que realizam tratamento no ambulatório de quimioterapia. Para o cálculo da amostra foi utilizada a amostragem probabilística simples, com nível de confiança de $90 \%$, e erro amostral de $7 \%$. Os critérios de exclusão da amostra foram: prontuários de pessoas que não estavam realizando tratamento quimioterápico, uma vez que estas são atendidas no mesmo ambulatório, porém, em acompanhamento pelas clínicas de dermatologia, reumatologia e gastroenterologia, sendo acometidas por outras condições crônicas de saúde.

Os resultados foram tabulados e organizados no Microsoft Excel, e foi utilizado o método de estatística descritiva, de frequências simples e percentual. As discussões dos resultados foram realizadas à luz da revisão de literatura, em consonância com as reflexões e críticas das autoras.

Este estudo foi aprovado pelo Comitê de Ética em Pesquisa do HUCFF, protocolo $n^{\circ} 130 / 10$, de acordo com a Resolução do Conselho Nacional de Saúde n 196/96.

\section{RESULTADOS}

No período do estudo foram analisados 87 dos 200 prontuários de usuários ativos no ambulatório de quimioterapia, o que corresponde a $43,5 \%$ do total. Destes, foi constatado que a maioria é do sexo feminino, com $59,7 \%$ de representatividade estatística.

No que se refere aos demais dados sociodemográficos, a maior parte dos usuários possui idade entre 51 a 60 anos, o que corresponde a um total de 25,3\%. Em seguida, a faixa etária de maior prevalência foi entre 61 a 70 anos, com 24,1\%; 12,6\% da amostra 
possuem de 71 a 80 anos; as faixas etárias de 31 a 40 anos e 41 a 50 anos tiveram a mesma representatividade, ou seja, $16,1 \%$ cada; $3,5 \%$ da amostra possuem de 21 a 30 anos; e 2,3\% de 81 a 90 anos.

Destaca-se a prevalência de casados, no caso de $59,8 \%$ da amostra, $31 \%$ dos usuários são solteiros ou divorciados e $9,2 \%$ viúvos, contudo, $81,6 \%$ residem com outras pessoas e apenas $18,4 \%$ moram sozinhos.

Com relação ao grau de escolaridade, $38 \%$ das pessoas possuem ensino fundamental incompleto; $26,4 \%$ ensino médio completo; $25,3 \%$ ensino fundamental completo; $4,6 \%$ ensino médio incompleto; no caso do ensino superior $2,3 \%$ possuem ensino incompleto, e o mesmo número ensino completo; e 1,1\% analfabeto.

No que tange à ocupação, em 64,4\% dos prontuários não havia registro com relação a esta variável. Dos 35,6\% restantes, $10,3 \%$ das pessoas são aposentadas, e as demais possuem algum tipo de atividade laborativa.

Na Tabela 1, foram reunidos os dados referentes ao perfil sociodemográfico.

Tabela 1: Perfil sociodemográfico das pessoas em tratamento quimioterápico no ambulatório do HUCFF. Rio de Janeiro, RJ, 2010 (N=87).

\begin{tabular}{|c|c|c|}
\hline Perfil sociodemográfico & $\mathbf{N}$ & $\%$ \\
\hline \multicolumn{3}{|l|}{ Sexo } \\
\hline Feminino & 52 & 59,7 \\
\hline Masculino & 35 & 40,3 \\
\hline \multicolumn{3}{|l|}{ Idade } \\
\hline 21-30 anos & 3 & 3,5 \\
\hline $31-40$ anos & 14 & 16,1 \\
\hline $41-50$ anos & 14 & 16,1 \\
\hline $51-60$ anos & 22 & 25,3 \\
\hline $61-70$ anos & 21 & 24,1 \\
\hline $71-80$ anos & 11 & 12,6 \\
\hline $81-90$ anos & 2 & 2,3 \\
\hline \multicolumn{3}{|l|}{ Estado civil } \\
\hline Casados & 52 & 59,8 \\
\hline Solteiros ou Divorciados & 27 & 31 \\
\hline Viúvos & 8 & 9,2 \\
\hline \multicolumn{3}{|l|}{ Estrutura Familiar } \\
\hline Residem acompanhados & 71 & 81,6 \\
\hline Residem sozinhos & 16 & 18,4 \\
\hline \multicolumn{3}{|l|}{ Educação } \\
\hline Ensino Fundamental Incompleto & 33 & 38 \\
\hline Ensino Médio Completo & 23 & 26,4 \\
\hline Ensino Fundamental Completo & 22 & 25,3 \\
\hline Ensino Médio Incompleto & 4 & 4,6 \\
\hline Ensino Superior Incompleto & 2 & 2,3 \\
\hline Ensino Superior Completo & 2 & 2,3 \\
\hline Analfabeto & 1 & 1,1 \\
\hline \multicolumn{3}{|l|}{ Ocupação } \\
\hline Sem registro desta variável & 56 & 64,4 \\
\hline Mantendo atividade laborativa & 22 & 25,3 \\
\hline Aposentados & 9 & 10,3 \\
\hline
\end{tabular}

Em relação ao perfil clínico das pessoas em tratamento quimioterápico ambulatorial o principal tipo de câncer apresentado foi o câncer de mama, acometendo $35,6 \%$ das mulheres, seguido do câncer de cólon e reto, com 20,7\%, próstata 17,3\%, cânceres hematológicos $6,9 \%$, pulmão $4,6 \%$, esôfago $3,4 \%$ e outros diversos com menor representatividade estatística cada, totalizando $11,5 \%$.

Quanto à proposta do tratamento, no momento da coleta de dados, 36,9\% estavam em tratamento paliativo, $28,7 \%$ adjuvante, $20,7 \%$ neo-adjuvante, $8 \%$ potencializadora e 5,7\% curativa. Com relação à fase da doença oncológica, em 11,5\% dos prontuários não foram encontrados registros acerca do estadiamento. Considerando a classificação TNM, sendo $T$ relativo ao tumor, $\mathrm{N}$ ao comprometimento de linfonodos e $\mathrm{M}$ à presença de metástases, constatou-se que $25,3 \%$ estavam no estadio avançado, ou seja, estadio IV. Porém, $57,5 \%$ tinham evidência de metástase. Utilizando outras classificações variadas para estadiamento foram identificados $46 \%$ da amostra. 
No que tange às principais queixas relacionadas ao tratamento quimioterápico as náuseas e vômitos foram as mais prevalentes, estando a primeira presente em $17,3 \%$ dos casos, e a segunda em $8 \%$. Dentre as demais, destacaram-se: febre em $7 \%$ dos casos, diarreia em $5,7 \%$ dos casos, dor abdominal, mucosite e disúria em $4,6 \%$ dos casos cada, e mialgia e dispneia em 3,4\% dos casos cada. Em 8\% dos prontuários os registros acusavam que as pessoas não apresentavam queixas. Outras queixas com menor representatividade sob o ponto de vista estatístico, como fadiga, tosse, inapetência, insônia, alopecia, cefaleia, disfagia, prurido e constipação somaram 16,1\%. Em 17,3\% dos prontuários não foram encontrados registros quanto às queixas relacionadas à quimioterapia.

No caso dos registros pelos profissionais de enfermagem das possíveis complicações na administração dos quimioterápicos, em 56,3\% dos prontuários constava que não ocorreram intercorrências durante o processo, e em $43,7 \%$ não foram encontrados registros sobre esta variável.

Quanto à via de administração dos quimioterápicos no ambulatório investigado, a predominante foi a via intravenosa, utilizada em $94,3 \%$ dos casos, seguida da via oral com $5,7 \%$.

Na Tabela 2 foram reunidos os dados referentes ao perfil clínico.

Tabela 2: Perfil clínico das pessoas em tratamento quimioterápico no ambulatório do HUCFF. Rio de Janeiro, RJ, 2010 (N=87).

\begin{tabular}{|c|c|c|}
\hline Perfil clínico & $\mathbf{N}$ & $\%$ \\
\hline \multicolumn{3}{|l|}{ Tipo de câncer } \\
\hline Câncer de mama & 31 & 35,6 \\
\hline Câncer de cólon e reto & 18 & 20,7 \\
\hline Câncer de próstata & 15 & 17,3 \\
\hline Cânceres hematológicos & 6 & 6,9 \\
\hline Câncer de pulmão & 4 & 4,6 \\
\hline Câncer de esôfago & 3 & 3,4 \\
\hline Outros & 10 & 11,5 \\
\hline \multicolumn{3}{|l|}{ Proposta de tratamento } \\
\hline Paliativo & 32 & 36,9 \\
\hline Adjuvante & 25 & 28,7 \\
\hline Neo-adjuvante & 18 & 20,7 \\
\hline Potencializado & 7 & 8 \\
\hline Curativa & 5 & 5,7 \\
\hline \multicolumn{3}{|l|}{ Estadiamento } \\
\hline Estadio II & 8 & 9,2 \\
\hline Estadio III & 7 & 8 \\
\hline Estadio IV & 22 & 25,3 \\
\hline Outras classificações & 40 & 46 \\
\hline Não registrado & 10 & 11,5 \\
\hline \multicolumn{3}{|l|}{ Evidência de metástase } \\
\hline Sim & 50 & 57,5 \\
\hline Não & 37 & 42,5 \\
\hline \multicolumn{3}{|l|}{ Principais queixas } \\
\hline Náuseas & 15 & 17,3 \\
\hline Vômito & 7 & 8 \\
\hline Febre & 6 & 7 \\
\hline Diarréia & 5 & 5,7 \\
\hline Dor abdominal & 4 & 4,6 \\
\hline Mucosite & 4 & 4,6 \\
\hline Disúria & 4 & 4,6 \\
\hline Mialgia & 3 & 3,4 \\
\hline Dispnéia & 3 & 3,4 \\
\hline Sem queixas & 7 & 8 \\
\hline Outras queixas & 14 & 16,1 \\
\hline Sem registro de queixas & 15 & 17,3 \\
\hline \multicolumn{3}{|l|}{ Complicações na administração } \\
\hline Não apresentaram & 49 & 56,3 \\
\hline Sem registro desta variável & 38 & 43,7 \\
\hline \multicolumn{3}{|l|}{ Via de administração } \\
\hline Endovenosa & 82 & 94,3 \\
\hline Oral & 5 & 5,7 \\
\hline
\end{tabular}




\section{DISCUSSÃO}

A maior prevalência do câncer nas mulheres vai ao encontro das estimativas do INCA para os anos de $2012 \mathrm{e}$ 2013, quando são esperados 260.640 casos novos de câncer para o sexo feminino. Destaca-se ainda, nesta população, o tumor mais incidente, ou seja, o de mama, que no âmbito nacional tem incidência estimada em 53.000 casos novos $^{(1)}$.

Considerando as contribuições do conhecimento do perfil sociodemográfico e clínico das pessoas assistidas para o gerenciamento das ações de enfermagem, destaca-se a possibilidade do estabelecimento de algumas relações para tomada de decisões. Cita-se, por exemplo, a relação entre a prevalência de mulheres e do câncer de mama com o procedimento da punção venosa periférica (PVP) para infusão de quimioterapia. Por conseguinte, a maior incidência do câncer na mama feminina requer cuidados de enfermagem específicos no momento da administração do quimioterápico pela via endovenosa, considerando a possibilidade de tratar-se de uma quimioterapia adjuvante, com eventual esvaziamento da rede ganglionar axilar. Neste caso é contra-indicada a PVP no membro afetado, devido ao risco de infecções e linfedema ${ }^{(10)}$.

Além disso, a mulher mastectomizada, ou em preparo para esta cirurgia, pode sofrer com a baixa autoestima e com o distúrbio da imagem corporal, o que requer atenção aos aspectos psicossociais, sendo fundamental que o enfermeiro a encaminhe para o atendimento psicológico e social, no contexto do ambulatório, sempre que necessário, assumindo a perspectiva de trabalho interdisciplinar. Esta postura vai ao encontro do princípio de integralidade do SUS, sendo uma vertente do cuidado em destaque na avaliação da qualidade de vida de pessoas tratadas contra o câncer ${ }^{(11)}$.

Em casos de impossibilidade de PVP, o enfermeiro deve participar da avaliação e da indicação de acessos venosos centrais, sendo o catéter central de inserção periférica (PICC) uma das possibilidades a serem examinadas, em especial diante da prevalência da via endovenosa para administração de quimioterapia, ou seja, em $94,3 \%$ dos casos. Este dispositivo tem sido considerado uma alternativa segura e eficaz, e sua inserção pelo enfermeiro requer que o mesmo seja treinado especificamente ${ }^{(12)}$. No Brasil, a Resolução $n^{\circ}$ $258 / 2001$ do $\operatorname{COFEN}^{(13)}$, permite a prática da inserção de catéter periférico central pelo enfermeiro devidamente treinado.

Diante da faixa etária prevalente, ou seja, entre 51 a 60 anos (25,3\%), é válido ressaltar que a mesma representa parte da população economicamente ativa e produtiva, e muitos usuários no ambulatório conciliam suas atividades laborativas durante o tratamento (25,3\%). A manutenção das atividades laborativas pode contribuir para a autoestima da pessoa, e na prática, deve contar com a flexibilidade nas ações de enfermagem, como por exemplo, no agendamento dos atendimentos, e nas orientações para o autocuidado.

Destaca-se na amostra do estudo elevado número de pessoas idosas, o que demanda planejamento, organização e avaliação do cuidado de enfermagem, considerando as respostas diferenciadas ao tratamento quimioterápico por parte das mesmas, bem como as limitações específicas relacionadas aos possíveis problemas sensoriais, de memória e para locomoção, por exemplo. As mudanças provocadas pela idade avançada podem afetar os efeitos e consequências do tratamento, aumentando a suscetibilidade às toxicidades. Dessa forma, o enfermeiro deve gerenciar o cuidado ao idoso atentando para as múltiplas necessidades, que conjugam domínios funcionais, fisiológicos, psicossociais e econômicos ${ }^{(14)}$.

As necessidades de cuidado do idoso, bem como das pessoas nas demais faixas etárias durante o tratamento contra o câncer podem ser melhor gerenciadas e atendidas a partir de uma estrutura familiar presente e fortificada ${ }^{(15)}$. Cabe considerar que $81,6 \%$ dos usuários residem com outras pessoas, $59,8 \%$ são casados, e que apenas $18,4 \%$ moram sozinhos, mas estes, mesmo nesta condição, podem compor outras redes sociais de apoio. A família deve ser considerada pelo enfermeiro como aliada para a implementação das medidas de cuidado, assim como deve ser atendida diante da manifestação de suas próprias necessidades.

O atendimento do binômio usuário/família perpassa pelo estabelecimento de comunicação eficaz e empática entre os mesmos e a equipe de saúde. Portanto, o enfermeiro deve valorizar a comunicação, já que dependendo da forma como a mesma é estabelecida, pode gerar interferências positivas ou negativas no processo diagnóstico-tratamento(16). A orientação adequada no decorrer do tratamento quimioterápico é essencial para o seu sucesso e prevenção de maiores 
danos. Tais orientações, considerando o contexto de vida das pessoas, o grau de escolaridade e o conhecimento relativo ao senso comum, podem compreender, por exemplo, o esclarecimento do que é o tratamento quimioterápico, seus eventos desejáveis e indesejáveis, diminuindo o déficit de conhecimento e aumentando a possibilidade do usuário desenvolver o controle aumentado do seu regime terapêutico.

Diante do elevado número de usuários em tratamento quimioterápico paliativo, ou seja, $36,9 \%$, e de sua relação com prognósticos sombrios, é importante que o enfermeiro compreenda os preceitos dos cuidados paliativos e gerencie o cuidado a partir de referenciais dinâmicos e flexíveis ${ }^{(17)}$. Além disso, ao longo do tratamento, a evolução da doença, considerando que 57,5\% dos usuários apresentam metástases, pode resultar na interrupção da quimioterapia, a partir da ponderação entre riscos e benefícios, ou ainda na piora da qualidade de vida ${ }^{(18)}$.

Cabe considerar, em especial, no caso das pessoas em fase avançada da doença oncológica, que o enfermeiro precisa organizar e preparar o ambiente para recebê-las de forma a promover o conforto. Logo, precisa ser levada em consideração, por exemplo, as condições físicas do ambiente para receber uma pessoa mais debilitada pela doença e que necessite permanecer na cadeira de rodas ou numa maca durante o tempo de permanência no ambulatório.

A predominância da proposta do tratamento quimioterápico paliativo compreende um dado extremamente significativo e que se relaciona com as problemáticas que envolvem as ações de prevenção primária e secundária, interferindo diretamente no tempo de sobrevida e na qualidade de vida dessas pessoas. Tais problemáticas relacionam-se com as iniquidades em saúde, que podem ser investigadas a partir de estudos que trazem determinantes sociais da saúde na população afetada por determinada doença ou condição(2). Sendo assim, um dos papéis sociais da enfermagem é intervir nos determinantes sociais dos processos saúde-doença, a partir do reconhecimento da realidade, assumindo como norteadores da prática social os princípios do SUS da equidade, da integralidade e da universalidade, e o respeito ao direito de cidadania das pessoas, como objeto de interesse social.

O conhecimento do perfil clínico das pessoas assistidas é capaz de orientar o enfermeiro na compreensão das bases terapêuticas utilizadas, bem como no raciocínio clínico frente aos problemas de ordem física apresentados. E quando relacionado com as questões sociodemográficas abrange a possibilidade do cuidado integral e contextualizado, em consonância com as necessidades das pessoas, favorecendo relações terapêuticas orientadas pelo respeito e por critério prognóstico mais realista.

Com relação às principais queixas, destacam-se as que se relacionam aos efeitos colaterais provocados pela ação indiscriminada dos quimioterápicos, em especial, no sistema digestório, como por exemplo: náuseas e vômitos; diarreia, dor abdominal e mucosite. Tais efeitos podem desencadear outros problemas capazes de interferir na condição clínica da pessoa no decorrer do tratamento, como comprometimento do estado nutricional, levando à caquexia ${ }^{(19)}$. Dessa forma, os efeitos colaterais devem ser evitados ou minimizados, já que interferem na qualidade de vida pessoal.

Realizar o registro das queixas é de vital importância, não só porque fomenta a continuidade, como permite a avaliação dos cuidados de enfermagem implementados. Nesse sentido, a prática de enfermagem organizada e sistematizada qualifica o cuidado, devendo ser operacionalizada por meio da implantação das etapas metodológicas do processo de enfermagem, contemplando o registro no prontuário. Em 17,3\% dos prontuários investigados não havia registro contemplando a variável relativa às principais queixas, o que está dentro do valor considerado satisfatório, ou seja, de até $20 \%{ }^{(20)}$.

Em contrapartida, ressalta-se a ausência de registros sobre as complicações na administração do quimioterápico em $43,7 \%$ dos prontuários. Em suma, nenhuma complicação foi registrada, uma vez que em $56,3 \%$ dos prontuários havia registro de procedimento realizado sem intercorrência. Este resultado encaminha para a necessidade de novos estudos, com o intuito de categorizar os eventos reconhecidos como complicação pelos membros da equipe de enfermagem, a considerar, por exemplo, a necessidade de realização de mais de uma PVP, seja por fragilidade capilar, por queixa de dor e ardência local ou por extravasamento, a partir, inclusive, do que é observado empiricamente no contexto investigado.

Além de todas as questões legais, registros precários dificultam a continuidade e a avaliação da assistência, 
como por exemplo, no caso da auditoria em enfermagem. É de responsabilidade dos enfermeiros o registro escrito completo das suas ações relativas ao cuidado, em prol da legitimação do trabalho da enfermagem ${ }^{(20)}$.

\section{CONCLUSÃO}

No que se refere ao perfil sociodemográfico das pessoas assistidas no ambulatório de quimioterapia investigado, destacam-se as seguintes características: a maioria do sexo feminino (59,7\%); grande número de pessoas idosas; $59,8 \%$ dos usuários são casados e $81,6 \%$ residem com outras pessoas; a maioria, ou seja, $38 \% \mathrm{com}$ ensino fundamental incompleto; e $25,3 \%$ mantendo atividade laborativa no decorrer do tratamento.

No caso do perfil clínico, destacam-se: a maioria com câncer de mama (35,6\%); 36,9\% com proposta de tratamento de quimioterapia paliativa, demonstrando ser percentual inferior comparado ao elevado número de pessoas com evidência de metástase, ou seja, 57,5\%, o que isoladamente classifica a doença em estadio avançado; principais queixas envolvendo o sistema digestório; sem relato de intercorrência na administração do quimioterápico em $56,3 \%$ dos prontuários, sendo a variável que expõe déficit no registro de enfermagem; e a via endovenosa como sendo a principal, prevalecendo em $94,3 \%$ dos casos.

As informações clínicas e não clínicas, baseadas em determinantes sociais da saúde, quando tratadas em concomitância, contribuem para diferentes aspectos gerenciais. No âmbito do gerenciamento do cuidado de enfermagem são capazes de guiar o processo de tomada de decisão e ação, considerando, por exemplo: cuidados específicos, como no caso da PVP; a organização do ambiente físico; a atenção à família; a observação da tolerância à toxicidade provocada pelo tratamento, principalmente nas pessoas idosas; bem como a evolução da doença, visando sempre a manutenção da qualidade de vida e do conforto da pessoa.

\section{REFERÊNCIAS}

1. Ministério da Saúde; Instituto Nacional de Câncer. Estimativa 2012 - Incidência de Câncer no Brasil. Rio de Janeiro: INCA; 2012. $118 \mathrm{p}$.

2. Geib LTC. Determinantes sociais de saúde do idoso. Ciênc. saúde coletiva. 2012;17(1):123-133.

3. Ministério da Saúde; Instituto Nacional de Câncer. Ações de enfermagem para controle do câncer: uma proposta de
Os benefícios do conhecimento do perfil decorrem, então, na maior possibilidade de prestação do cuidado integral em consonância com a realidade de cada indivíduo, respeitando suas particularidades, bem como adequando as ações de acordo com as respostas individuais, de forma personalizada, contextualizada e humanizada.

A amplitude das ações do gerenciamento do cuidado de enfermagem, que incluem o planejamento, a organização, a supervisão, a execução e a avaliação de todas as atividades desenvolvidas na assistência à pessoa, demandam a visão ampliada por parte do enfermeiro, o desenvolvimento de competências e habilidades múltiplas que valorizam a comunicação, assim como, o trabalho na perspectiva interdisciplinar.

Dessa forma, o conhecimento do perfil sociodemográfico e clínico das pessoas assistidas subsidiam as ações do enfermeiro nas principais dimensões do seu processo de trabalho, ou seja, gerencial e assistencial. Esta afirmativa decorre da utilidade de tais informações, por exemplo, para o gerenciamento dos recursos materiais, o controle de equipamentos, o custo e escala de pessoal, o estabelecimento das relações interpessoais e execução das atividades educacionais, e por fim, o desenvolvimento de práticas para atender as necessidades das pessoas e seus familiares.

Considerando a complexidade do tema abordado, deve-se destacar que não houve a pretensão de se esgotar o assunto, e sim colaborar com uma parcela do conhecimento da enfermagem nesta área de atuação. Além disso, há encaminhamentos para a necessidade de novas investigações científicas, considerando a escassez de estudos na literatura atual sobre a temática apresentada neste estudo, bem como relativa à qualidade do registro de enfermagem no que concerne ao reconhecimento de complicações ou intercorrências no decorrer da administração do quimioterápico, uma vez que em $43,7 \%$ não foram identificados registros sobre esta variável.

integração ensino-serviço. $3^{\text {a }}$ ed. Rio de Janeiro: INCA; 2008. $628 \mathrm{p}$.

4. Conselho Federal de Enfermagem. Resolução nº 210 de 1998. Dispõe sobre a atuação dos profissionais de enfermagem que trabalham com quimioterápico antineoplásicos. Rio de Janeiro (Brasil); 1998. 
5. Christovam BP, Porto IS, Oliveira DC. Gerência do cuidado de enfermagem em cenários hospitalares: a construção de um conceito. Rev Esc Enferm USP. 2012;46(3):734-41.

6. Mansano-Schlosser TC, Ceolim MF. Qualidade de vida de pacientes com câncer no período de quimioterapia. Texto Contexto Enferm. 2012;21(3):600-7.

7. Villegas CR, Chacón JA, Cardona JP, Correa LA. Perfil clínico e epidemiológico de los pacientes con cáncer tratados en una institución de tercer nivel. Colomb Med. 2012;43(1):11-18. 8. Ministério da Saúde. Portaria $n^{\circ} 2.439$ de 08 de dezembro de 2005. Institui a Política Nacional de Atenção Oncológica a ser implantada em todas as unidades federadas, respeitadas as competências das três esferas de gestão. Brasília (Brasil): Ministério da Saúde; 2005.

9. Gil AC. Métodos e técnicas de pesquisa social. $6^{a}$ ed. São Paulo: Atlas; 2008

10. Soares CR, Almeida AM, Gozzo TO. A avaliação da rede venosa pela enfermagem em mulheres com câncer ginecológico durante o tratamento quimioterápico. Esc Anna Nery. 2012;16(2):240-6.

11. Jorge LS, Silva SR. Avaliação da qualidade de vida de portadores de câncer ginecológico, submetidas à quimioterapia antineoplásica. Rev Latino-Am. Enfermagem. 2010;18(5):849855.

12. Bellesi S, Chiusolo P, De Pascale G, Pittiruti M, Scoppettuolo $G$, Metafuni E, et al. Peripherally inserted central catheters (PICCS) in the management of oncohematological patients submitted to autologous stem cell transplantation. Support Care Cancer. 2013;21(2):531-5.

13. Conselho Federal de Enfermagem. Resolução $n^{\circ} 258 / 2001$ do COFEN. Resenha: Inserção de catéter periférico central, pelos enfermeiros. Rio de Janeiro (Brasil); 2001.

14. Johnson M. Chemotherapy treatment decision making by professionals and older patients with cancer: a narrative review of the literature. Eur J Cancer Care. 2012;21(1):3-9.

15. Gómez CEP, Sánchez LVG, Enriquez JI. Experiencias de vida en mujeres con cáncer de mama en quimioterapia. Rev Colomb Psiquiatr. 2011;40(1):65-84.

16. Albuquerque ZBP, Tavares SBN, Manrique EJC, Souza ACS, Neves HCC, Valadares JG et al. Atendimento pelo SUS na percepção de mulheres com lesões de câncer cervicouterino em Goiânia-GO. Rev. Eletr. Enf. [Internet]. 2011 [cited 2012 Jan 20];13(2):239-49. Available from: http://www.fen.ufg.br/revista/v13/n2/v13n2a10.htm

17. Silva MM, Moreira MC. Desafios à sistematização da assistência de enfermagem em cuidados paliativos oncológicos: uma perspectiva da complexidade. Rev. Eletr. Enf. [Internet]. 2010 [cited 2011 July 15];12(3):483-90. Available from: http://www.fen.ufg.br/revista/v12/n3/v12n3a10.htm 18. Mayrbäurl B, Wintner LM, Giesinger JM,

Himmelfreundpointner T, Burgstaller S, Holzner B, et al. Chemotherapy line-associated differences in quality of life in patients with advanced cancer. Support Care Cancer. 2010;20(10):2399-405.

19. Calixto-Lima L, Andrade EM, Gomes AP, Geller M, SiqueiraBatista R. Dietetic management in gastrointestinal complications from antimalignant chemoterapy. Nutr Hosp. 2012;27(10):65-75.

20. Silva JA, Grossi ACM, Haddad MCL, Marcon SS. Avaliação da qualidade das anotações de enfermagem em unidade semiintensiva. Esc Anna Nery. 2012;16(3):577-82.

Artigo recebido em 27/09/2012.

Aprovado para publicação em 23/05/2013.

Artigo publicado em 30/09/2013. 